\title{
YAW SENSOR DESIGN OPTIMIZATION USING FINITE ELEMENT METHOD
}

\author{
George Q. Jiang \\ Delphi Delco Electronics Systems, MS Fab A, P.O. Box 9005, \\ Kokomo, IN 46904-9005
}

\begin{abstract}
Delphi Delco Electronics Systems, in collaboration with the Delphi R\&D Center, has developed a surface-micromachined angular rate (yaw) sensor for automotive applications. This sensor is based on the same principle used for the vibrating shell gyroscope. The sensing element consists of a combined ring and comb structure, which is supported by eight springs attached to a center hub. Modal analysis has been performed on the sensing element to study the mode shapes of different designs using the finite element method (FEM). It was discovered that mode shapes were distorted due to the asymmetry of the supporting spring geometry in previous designs. The distorted mode shape limited the sensor's performance. The finite element method was also used to optimize a novel spring design to restore the sensing element's mode shape. The optimized design has eliminated the sensor stiction at hight temperature, reduced the total harmonic distortion (THD), simplified application specific integrated circuit (ASIC) design and sensor calibration, and improved sensor performance and yield.
\end{abstract}

\section{INTRODUCTION}

The surface-micromachined, vibrating ring angular rate sensor is fabricated with a electroforming process that allows for the inclusion of large amounts of on-chip circuitry with the sensing element on the same die. Wafer-level vacuum packaging has also been developed to further reduce the cost [1]. It is the low cost that enables wider automotive applications including yaw control, rollover protection, and tum-by-tum navigation presently implemented only in the luxury-vehicle market.

The sensing element is based on the principle of the shell vibrating in its fundamental flexural mode analyzed by $\mathrm{G} . \mathrm{H}$. Bryan in 1890 [2]. In this mode of vibration, the lip of the shell (or the rim of a ring) vibrates in an elliptical shape that has two nodal diameters. The four points on the ring that have no radial deflection are called nodes, and the four points with maximum radial deflection, located $45^{\circ}$ from the nodes, are called antinodes (Figure 1). When the device is rotated, the Coriolis force causes the vibrating pattern to rotate and the amplitude of vibration at the defined nodal points to increase. To measure angular rate of rotation, the vibration signal at the nodes is fed back to reduce the amplitude, thereby-maintaining a stationary pattern with nodes at the defined locations. The signal is proportional to the angular rate and is used as the output [3].

This principle has been successfully applied to a navigationquality gyroscope developed by Delco Systems Operations for many years [4]. To simplify the manufacturing process and to reduce the cost, a surface-micromachined, vibrating-ring angular rate sensor was developed at the Delphi R\&D Center (formerly the GM Research Center). Delphi Delco Elcctronics Systems has improved the design further and made it a manufacturable, lowcost product. The sensing element consists of three structures: the ring itself; the eight combs attached to the ring at the antinodes and nodes; and eight support springs with one end attached to the ring and the other end attached to the center hub anchored on the substrate (Photo 1). The interdigitated combs form the parallel plate capacitors that are used for either driving the ring to resonance or detecting the rate signal. Different spring designs, such as a semicircle (C-shaped) or alternating, conjoined semicircles (S-shaped) [not shown], were used to support the ring and comb.

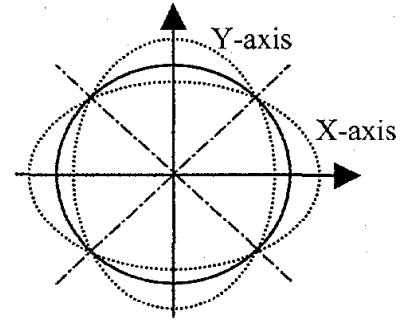

(a)

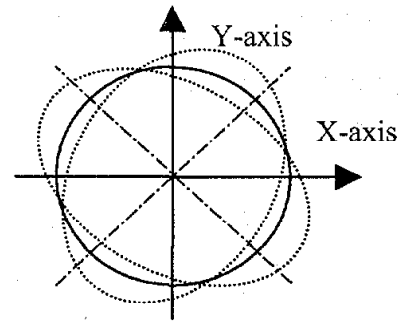

(b)
Figure 1. Mode shape of an ideal ring at flexural mode. (a) vibration pattern at station (b) vibration pattern at rotation

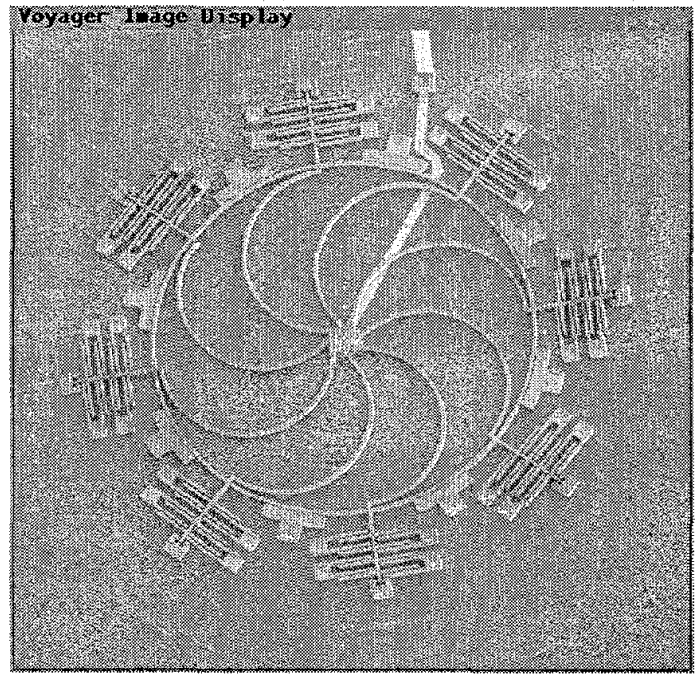

Photo1. Resonating ring-comb angular rate sensor

\section{ANALYSIS AND RESULTS}

It was very difficult to calibrate the prior-generation yaw sensor to a scale factor variation of less than $3 \%$ over the automotive operating temperature range $\left(-40\right.$ to $\left.85^{\circ} \mathrm{C}\right)$. The sensor also showed large, asymmetrical THD over the operating temperature range. At high temperature, the combs touched and shorted to the fixed electrodes, resulting in failure. To investigate the source of the problem, analyses were performed on the mode 
shape of the spring-supported, vibrating-ring yaw sensors and the results were compared with the mode shape of the ideal ring.

\section{The Mode Shape of an Ideal Ring}

The mode shape of an ideal ring can be expressed as

$$
\begin{aligned}
& U_{r}=A_{m} \times \cos (2 \theta) \\
& U_{\theta}=0.5 A_{m} \times \sin (2 \theta)
\end{aligned}
$$

where $U_{r}$ is the radial deflection of the ring

$U_{\theta}$ is the tangential motion of the ring

$A_{m}$ is the maximum amplitude of the deflection

$\theta$ is the angle of the ring with respect to the $x$-axis

The mode shape of an ideal ring can also be plotted as in Figure 2. It can be scen that at antinodes, which are located at 0 (360), 90, 180 and 270 degrees, the ring has maximum radial deflection while the tangential motion is zero. On the other hand, at the nodes, which are located at 45, 135, 225 and 315 degrees, the ring has maximum tangential motion but no radial deflection.

The FEM is also used to obtain the mode shape of an ideal vibrating-ring. The results are the same as shown in Figure 2.

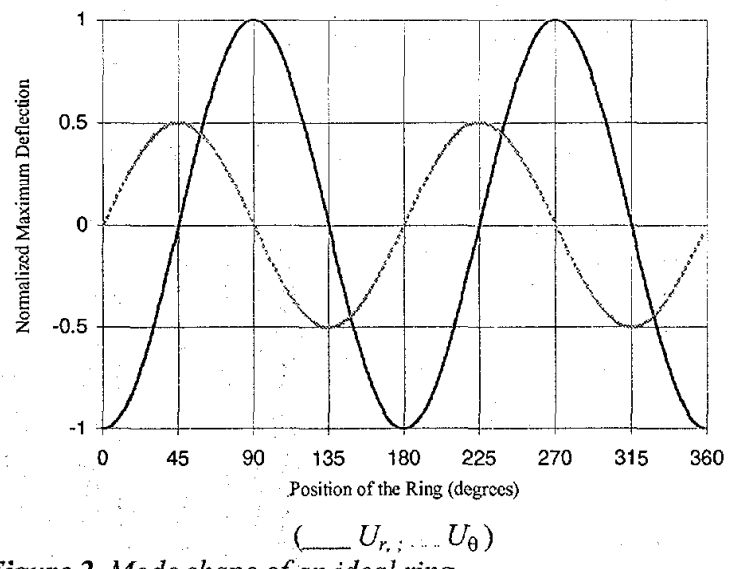

Figure 2. Mode shape of an ideal ring

\section{The Mode Shape of a Ring Supported by Springs}

Due to the complexity of the structure, no analytical mode shape solution is available for the springs-supported ring-comb sensing element. The FEM program, ANSYS $\otimes$, is used to perform modal analysis on these structures. The element SHELL43 is used to build the model. Nodal displacement is extracted from the simulation result file and analyzed. The mode shape of the ringcomb structure supported by $\mathrm{C}$-shaped springs is plotted in Figure 3.

It is clear from Figure 3 that the antinodes, where no tangential motion exists, and the nodes, where no radial motion exists, are no longer 45 degrees apart. Furthermore, the mode shape is not symmetrical about either antinodes or nodes as it is for an ideal ring. In other words, if the nodes are not aligned with the sensing electrodes at $45,135,225$, and 315 degrees, the radial motion will cause capacitance change or offset even when the yaw sensor is not subjected to angular rotation. If the balance mechanism is used to align the nodes with sensing electrodes located at 45, 135, 225, and 315 degrees, the antinodes will be shifted away. The electrodes located at $0,90,180$, and 270 degrees will see tangential motion in addition to radial motion. This additional motion distorts the signal at the pickup electrodes, increases the $2 \times$ frequency signal and therefore the total harmonic distortion (THD), which makes the task of calibrating the gyroscope's performance over a temperature range difficult. The tangential motion of the ring also causes comb rocking and even failure due to combs touching and becoming stuck to the sensing electrodes.

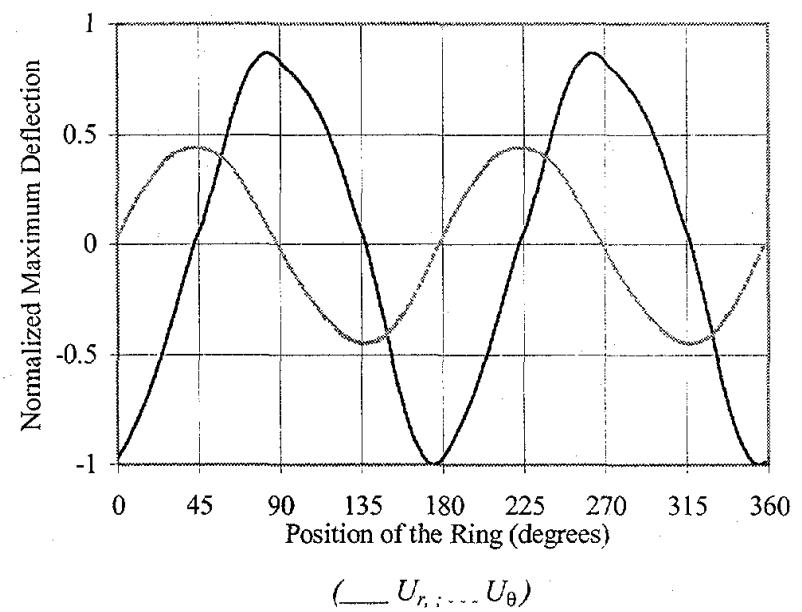

Figure 3. Mode shape of a ring supported by C-shaped springs

The mode shape of the resonance ring yaw sensors, supported by alternating, conjoined semicircle, S-shaped springs, has also been analyzed using ANSYSB. The simulation shows similar mode-shape distortion.

\section{DESIGN OPTIMIZATION}

The cause of mode-shape distortion aforementioned is the asymmetry of the previous spring designs, such as the C-shaped or the S-shaped spring configurations. To miniaturize the device and maintain low resonance frequency, however, a simple and asymmetrical shape of springs is often the choice. To minimize the mode-shape distortion, a novel-shaped spring system was invented which contains eight double-semicircle, S-shaped springs with two different radii (Photo 2). The ratio of the radius of the large semicircle to the radius of the small semicircle is optimized so that the mode-shape distortion will be minimized. With eight combs attached to the ring, the ratio of the radius of the large semicircle to the radius of the small semicircle is chosen as $3.28: 1$. Figure 4 shows the simulation results of the mode shape of a ring supported by the optimized springs.

It can be seen that in the optimized supporting spring design, the tangential motion at antinodes and the radial deflection at nodes are minimized. The mode shape of the resonating yaw sensor has been restored.

\section{SIGNIFICANT IMPACT}

Using the optimized supporting-spring design, the high temperature stiction problem has been eliminated. The THD over temperature has been reduced by $50 \%$ (Figure5). The most significant impact is that the combination of the new spring design and a simplified and matched ASIC design has dramatically increased the yield of the high-performance yaw sensor. The optimized design meets the requirement of a scale factor variation less than $3 \%$ over automotive operating temperatures with a greatly improved yield. 


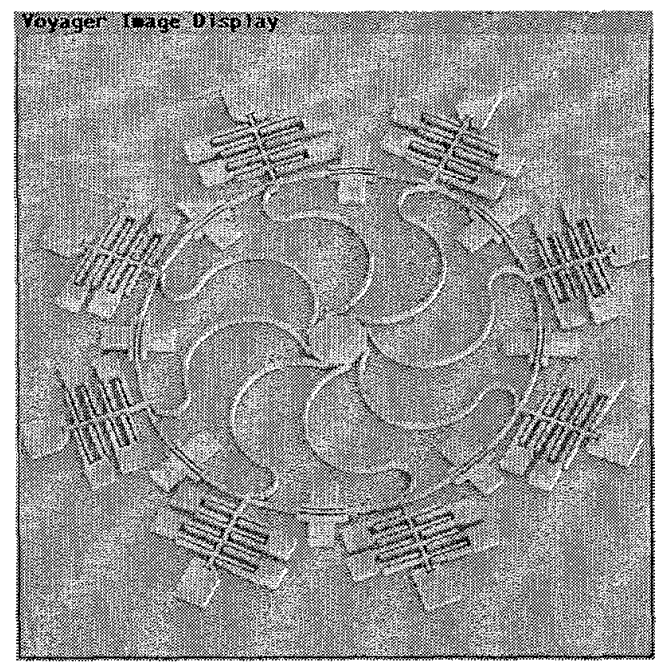

Photo 2. Resonance ring yaw sensor supported by the optimized springs

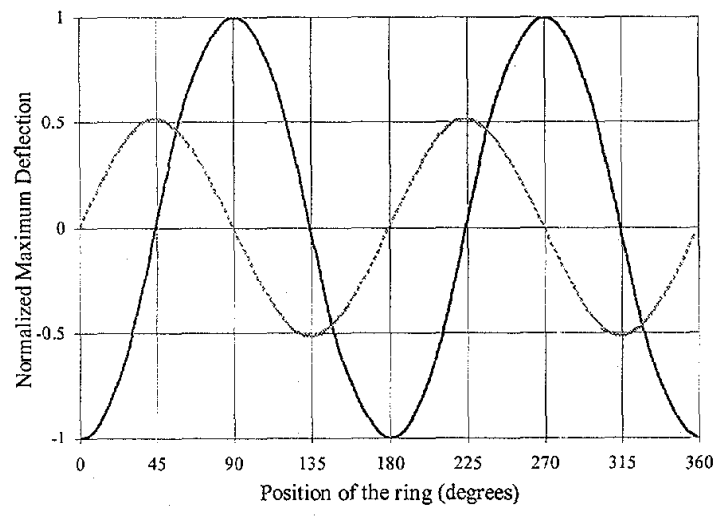

$\left(U_{r, ; \ldots} U_{\theta}\right)$

Figure 4. Mode shape of a ring supported by optimized springs

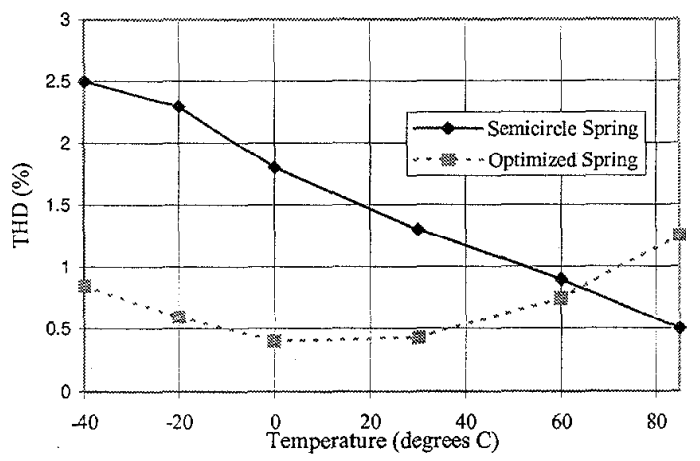

Figure 5. THD of different spring designs over operating temperature

\section{CONCLUSIONS}

The finite element method has been used to analyze the mode shape of the resonating-ring angular rate sensor. It is found that the mode shape of prior-generation sensors was distorted by the asymmetry of C- and S-shaped supporting springs. Modeshape distortion caused undesired radial deflection at the nodes and tangential motion at the antinodes. This, in turn, caused the following: large asymmetry THD over the operating temperature range, sensing structures shorted at high temperature, difficulty in calibration, and low yield.

The finite element method has also been used to develop a novel spring design in which each spring is composed of two semicircle-shaped springs with different radii. The ratio of the two radii is optimized so that the distortion of the mode shape of the vibrating ring is minimized. The new design has eliminated the sensor stiction at high temperature, minimized the THD, simplified the calibration process, and greatly improved the yield of the highperformance yaw sensor.

\section{ACKNOWLEDGMENTS}

The author would like to thank Mr. Tim Vas and Dr. Doug Sparks for their encouragement and support of this work. The author also appreciates the excellent efforts of Mr. Mike Chia, Mr. Jack Johnson and Mr. John Frazee in testing, and Dr. Seyed Zarabadi in ASIC design revision to match the sensing element.

\section{REFERENCES}

1. S. Zarabadi, T.Vas, D. Sparks, J. Johnson, Q. Jiang, M. Chia, E. Borzabadi, A Resonance Comb/Ring Angular Rate Sensor Vacuum Packaged Via Wafer Bonding, Spring SAE Tech. Proc., No. 1999-01-1043.

2. G. H. Bryan, On a Revolving Cylinder or Bell, Proceedings of the Royal Society (London), p. 47, 1890.

3. M. Putty, K. Najafi, A Micromachined Vibrating Ring Gyroscope, Solid-State Sensors and Actuators Workshop, Hilton Head, South Carolina, USA, pp. 213-320, June, 1994.

4. E. Loper, D. Lynch, Projected System Performance Based on Recent HRG Test Results, Proc. IEEE $5^{\text {th }}$ Digital Avionics System Conf. Seattle, Washington, USA, pp. 18.1.1-18.1.6, 1983. 